\title{
Christoffel and Fibonacci Tiles
}

\author{
Alexandre Blondin-Massé, Srečko Brlek ${ }^{\star \star}$, Ariane Garon, and Sébastien Labbé \\ Laboratoire de Combinatoire et d'Informatique Mathématique, \\ Université du Québec à Montréal, \\ C.P. 8888 Succursale "Centre-Ville", Montréal (QC), Canada H3C 3P8 \\ blondin_masse.alexandre@courrier.uqam.ca, brlek.srecko@uqam.ca, \\ garon.ariane@courrier.uqam.ca, labbe.sebastien@courrier.uqam.ca
}

\begin{abstract}
Among the polyominoes that tile the plane by translation, the so-called squares have been conjectured to tile the plane in at most two distinct ways (these are called double squares). In this paper, we study two families of tiles : one is directly linked to Christoffel words while the other stems from the Fibonacci sequence. We show that these polyominoes are double squares, revealing strong connections between discrete geometry and other areas by means of combinatorics on words.
\end{abstract}

Keywords: Polyomino, tiling, Christoffel word, Fibonacci sequence.

\section{Introduction}

In a discussion following the presentation of X. Provençal at the DGCI 2006 conference held in Szeged [1, E. Andres asked if there were a description of tesselations of the plane with tiles whose borders were discrete segments. That was the starting point of an investigation revealing interesting connections between discrete geometry, combinatorics on words, Lindenmayer systems, cristallography and number theory. The basic object of study is the ubiquitous polyomino, widely studied in the literature for having connections in numerous fields, whose list would be needless for our purpose.

There are different types of polyominoes and here, by polyomino we mean a finite union of unit lattice squares (pixels) in

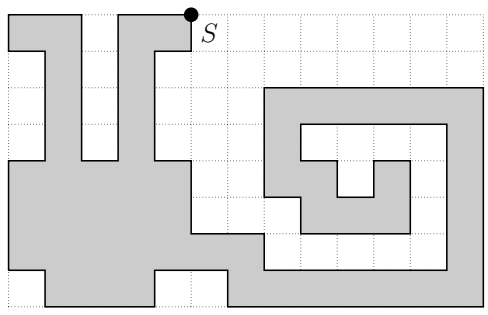

Fig. 1. A snail-shaped polyomino the discrete plane whose boundary is a simple closed path. In particular, a polyomino is simply connected (without holes), and its boundary is simple (does not cross itself). Paths are conveniently encoded by words on the alphabet $\{a, \bar{a}, b, \bar{b}\}$, representing the elementary grid steps $\{\rightarrow, \leftarrow, \uparrow, \downarrow\}$. For instance, starting from $S$ in counterclockwise way, the

\footnotetext{
* With support of the NSERC.

$\star \star$ Corresponding author. 
boundary $b(P)$ of the polyomino $P$ in Figure 1 is coded by the word

$$
w=\bar{a}^{2} \bar{b}^{4} \bar{a} b^{4} \bar{a}^{2} \bar{b} a \bar{b}^{3} \bar{a} \bar{b}^{3} a \bar{b} a^{3} b a^{2} \bar{b} a^{7} b^{6} \bar{a}^{6} \bar{b}^{3} a \bar{b} a^{3} b^{2} \bar{a} \bar{b} \bar{a} b \bar{a} b a^{4} \bar{b}^{4} \bar{a}^{5} b \bar{a}^{2} b^{2} \bar{a} b^{3} a b .
$$

Observe that we may consider the words as circular which avoids a fixed origin.

The perimeter of a polyomino $P$ is the length of its boundary words and is of even length. The problem of deciding if a given polyomino tiles the plane by translation goes back to Wisjhoff and Van Leeuven [2] who coined the term exact polyomino for these. Beauquier and Nivat [3] gave a characterization stating that the boundary $b(P)$ of an exact polyomino $P$ satisfies the following (not necessarily in a unique way) factorization

$$
b(P)=A \cdot B \cdot C \cdot \widehat{A} \cdot \widehat{B} \cdot \widehat{C}
$$

where at most one of the variables is empty, $\hat{\cdot}=\tau_{0} \widetilde{\cdot}, \widetilde{r}$ is the usual reversal operation and $\bar{r}$ is the morphism defined by $a \leftrightarrow \bar{a}$ and $b \leftrightarrow \bar{b}$ (see next section). Hereafter, this condition is referred as the BN-factorization.

For example, the polyomino in Figure 2 is exact, has semi-perimeter 14 and its boundary may be factorized as

$a b a b a \cdot b b a \bar{b} a \cdot b b \bar{a} b \cdot \bar{a} \bar{b} \bar{a} \bar{b} \bar{a} \cdot \bar{a} b \bar{a} \bar{b} b \cdot \bar{b} a \overline{b b}$. Polyominoes having a factorization with $A, B$ and $C$ nonempty are called pseudo-hexagons. If one of the variable is empty, they were called pseudo-squares, and from now on we call them squares. It has been shown in 4 that there exist polyominoes admitting an arbitrary number of distinct non trivial factorizations as pseudo-hexagons. The case is dif-

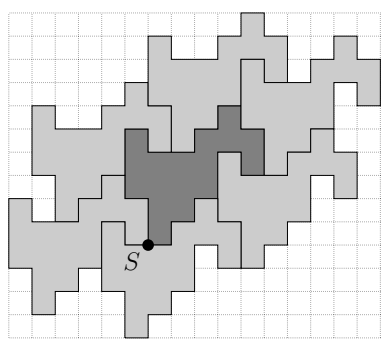

Fig. 2. A pseudo-hexagon ferent for squares. Indeed, it was conjectured in [4] that a square polyomino has at most two distinct non trivial factorizations.

Polyominoes admitting two distinct factorizations as squares (see Figure 3 for instance) are called double squares. A brute-force search based on Equation (11) can enumerate double squares exhaustively but as they have very specific structural properties, an efficient way to generate them has a greater interest. Moreover, some conjectures (e.g. related to palindromes [4]) could be solved by a complete de-

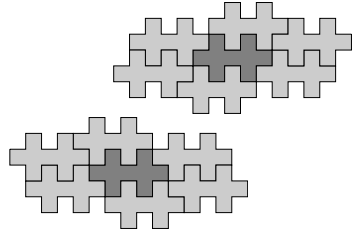

Fig. 3. A double square and its two square tilings scription of double squares. In this paper we use a combinatorial approach, relying on efficient techniques [15], to construct two classes of double square polyominoes. Those two families, interesting in themselves, are important for the zoology because they describe entirely the table of small double squares available in [4. The first is composed of Christoffel tiles, those for which the boundary word is composed of crenellated versions of two digitized segments (answering partially to E. Andres' question), for which a characterization is provided (Theorem 2). The second is built on the Fibonacci recurrence: a special 
subclass of Fibonacci tiles is completely described (Theorem 3), and we describe four more derived classes of squares polyominoes. Finally, this study reveals new connections with Lindenmayer systems and number theory among others.

\section{Preliminaries}

The usual terminology and notation on words is from Lothaire 6. An alphabet $\Sigma$ is a finite set whose elements are called letters. A finite word $w$ is a sequence of letters, that is, a function $w:\{1,2, \ldots, n\} \rightarrow \Sigma$, where $w_{i}$ is the $i$-th letter, $1 \leq i \leq n$. The length of $w$, denoted by $|w|$, is given by the integer $n$. The unique word of length 0 is denoted $\varepsilon$, and the set of all finite words over $\Sigma$ is denoted $\Sigma^{*}$. The free monoid $\Sigma^{*}$ is the set of all finite words over $\Sigma$, and $\Sigma^{\geq k}$ is the set of words of length at least $k$. The reversal $\widetilde{w}$ of $w=w_{1} w_{2} \cdots w_{n}$ is the word $\widetilde{w}=w_{n} w_{n-1} \cdots w_{1}$. Words $p$ satisfying $p=\widetilde{p}$ are called palindromes. The set of all palindromes over $\Sigma$ is denoted $\operatorname{Pal}\left(\Sigma^{*}\right)$. A word $u$ is a factor of another word $w$ if there exist $x, y \in \Sigma^{*}$ such that $w=x u y$. We denote by $|w|_{u}$ the number of times that $u$ appears in $w$. Two words $u$ and $v$ are conjugate if there are words $x$ and $y$ such that $u=x y$ and $v=y x$. In that case, we write $u \equiv v$. Clearly, $\equiv$ is an equivalence relation. Given two alphabets $\Sigma_{1}$ and $\Sigma_{2}$, a morphism is a function $\varphi: \Sigma_{1}^{*} \rightarrow \Sigma_{2}^{*}$ compatible with concatenation, that is, $\varphi(u v)=\varphi(u) \varphi(v)$ for any $u, v \in \Sigma_{1}^{*}$. It is clear that a morphism is completely defined by its action on the letters of $\Sigma_{1}$.

Paths on the square lattice. The notation of this section is partially adapted from [5]. A path in the square lattice, identified as $\mathbb{Z} \times \mathbb{Z}$, is a polygonal path made of the elementary unit translations

$$
a=(1,0), \bar{a}=(-1,0), b=(0,1), \bar{b}=(0,-1) .
$$

A finite path $w$ is therefore a word on the alphabet $\mathcal{F}=\{a, \bar{a}, b, \bar{b}\}$, also known as the Freeman chain code [78] (see [9] for further reading). Furthermore, we say that a path $w$ is closed if it satisfies $|w|_{a}=|w|_{\bar{a}}$ and $|w|_{b}=|w|_{\bar{b}}$. A simple path is a word $w$ such that none of its factor is a closed path. A boundary word is a closed path such that none of its proper factors is closed. Finally, a polyomino is a subset of $\mathbb{Z}^{2}$ contained in some boundary word. For instance, for the paths
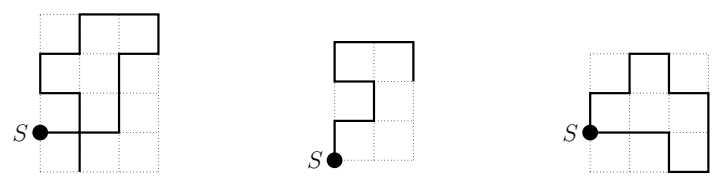

Fig. 4. Paths in the discrete plane: (a) arbitrary, (b) simple (c) closed

represented in Figure 4, the corresponding Freeman chain code is respectively
(a) $p_{1}=a b \bar{a} b a b a a \bar{b} \bar{a} \overline{b b} \bar{a} \bar{b}$
(b) $p_{2}=b a b \bar{a} b a a \bar{b}$
(c) $p_{3}=b a b a \bar{b} a \overline{b b} \bar{a} b \overline{a a}$. 
On the square grid, a path describes a sequence of basic movements in the left (L), right (R), forward (F) and backward (B) directions. Each pair of letters in $\mathcal{F}$ is associated to a movement on the alphabet $\mathcal{R}=\{\mathrm{L}, \mathrm{R}, \mathrm{F}, \mathrm{B}\}$ by a function $g: \mathcal{F}^{2} \rightarrow \mathcal{R}$ defined by

$$
g(u)= \begin{cases}\mathrm{L} & \text { if } u \in V_{\mathrm{L}}=\{a b, b \bar{a}, \bar{a} \bar{b}, \bar{b} a\}, \\ \mathrm{R} & \text { if } u \in V_{\mathrm{R}}=\{b a, a \bar{b}, \bar{b} \bar{a}, \bar{a} b\}, \\ \mathrm{F} & \text { if } u \in V_{\mathrm{F}}=\{a a, \overline{a a}, \overline{b b}, b b\}, \\ \mathrm{B} & \text { if } u \in V_{\mathrm{B}}=\{a \bar{a}, \bar{a} a, b \bar{b}, \bar{b} b\},\end{cases}
$$

that can be extended to a derivative function $\partial: \mathcal{F}^{\geq 1} \rightarrow \mathcal{R}^{*}$ by setting

$$
\partial w= \begin{cases}\prod_{i=2}^{n} g\left(w_{i-1} w_{i}\right) & \text { if }|w| \geq 2 \\ \varepsilon & \text { if }|w|=1\end{cases}
$$

where $n$ is the length of the word $w$ and the product is the concatenation. For example, if $p_{2}=b a b \bar{a} b a a \bar{b}$ as defined above then $\partial p_{2}=$ RLLRRFR.

Note also that each path $w \in \mathcal{F}^{\geq 1}$ is completely determined, up to translation, by its initial step $\alpha \in \mathcal{F}$ and a word $y$ on the alphabet $\mathcal{R}=\{\mathrm{L}, \mathrm{R}, \mathrm{F}, \mathrm{B}\}$. Thus, we use calculus notation and we introduce a function $\int_{\alpha}: \mathcal{R}^{*} \rightarrow \mathcal{F}^{\geq 1}$ defined recursively by

$$
\int_{\alpha} y= \begin{cases}\alpha & \text { if }|y|=0, \\ \alpha \int_{\beta} y^{\prime} & \text { if }|y| \geq 1,\end{cases}
$$

where $\beta \in \mathcal{F}$ is the letter such that $\alpha \beta \in V_{x}$ and $y=x y^{\prime}$ with $x \in \mathcal{R}$. For example, if $y=$ RLLRRFR, then $\int_{b} y=b a b \bar{a} b a a \bar{b}=p_{2}$. The next lemma gives some easily established statements and shows how both functions $\partial$ and $\int$ behave naturally.

Lemma 1. Let $w, w^{\prime} \in \mathcal{F}^{*}, y, y^{\prime} \in \mathcal{R}^{*}, \alpha \in \mathcal{F}$ and $x \in \mathcal{R}$. Then

(i) $\int_{w_{1}} \partial w=w$ and $\partial \int_{\alpha} y=y$, where $w_{1}$ is the first letter of $w$.

(ii) $\partial\left(w w^{\prime}\right)=\partial w \cdot g\left(w_{n} w_{1}^{\prime}\right) \cdot \partial w^{\prime}$ and $\int_{\alpha} y x y^{\prime}=\int_{\alpha} y \int_{\beta} y^{\prime}$, where $w_{n}$ is the last letter of $w, w_{1}^{\prime}$ is the first letter of $w^{\prime}$ and $\beta$ is the last letter of $\int_{\alpha} y x$.

Note that $|\partial w|=|w|-1$ and $\left|\int_{\alpha} y\right|=|y|+1$. In some situations, for example when $w$ represents a circular word, this is not convenient. For that reason we introduce two auxiliary functions. The first $\stackrel{\circ}{\partial}: \mathcal{F}^{*} \rightarrow \mathcal{R}^{*}$ is defined by

$$
\stackrel{\circ}{\partial} w= \begin{cases}\varepsilon & \text { if }|w|=0, \\ \partial w w_{1} & \text { if }|w| \geq 1,\end{cases}
$$

where $w_{1}$ is the first letter of $w$. The second is $\oint_{\alpha}: \mathcal{R}^{*} \rightarrow \mathcal{F}^{*}$, defined as follows : if $\beta$ is the last letter of $\int_{\alpha} y$, then $\oint_{\alpha} y$ is the word such that $\oint_{\alpha} y \cdot \beta=\int_{\alpha} y$. It is clear that $\oint_{w_{1}} \stackrel{\circ}{\partial} w=w$ and $\stackrel{\circ}{\partial} \oint_{\alpha} y=y$ for all $w \in \mathcal{F}^{*}$ and $y \in \mathcal{R}^{*}$.

In [5], the authors also introduced a valuation function $\Delta$ defined on $\mathcal{R}^{*}$ by $\Delta(y)=|y|_{\mathrm{L}}-|y|_{\mathrm{R}}+2|y|_{\mathrm{B}}$ as well as on $\mathcal{F}^{\geq 1}$ by setting $\Delta(w)=\Delta(\partial w)$. This 
valuation is nothing but the winding number.

Transformations. Some useful transformations on $\mathcal{F}^{*}$ are rotations by an angle $k \pi / 2$ and reflections with respect to axes of angles $k \pi / 4$, where $k \in \mathbb{N}$. The rotation of angle $\pi / 2$ translates merely in $\mathcal{F}$ by the morphism:

$$
\rho: a \mapsto b, b \mapsto \bar{a}, \bar{a} \mapsto \bar{b}, \bar{b} \mapsto a .
$$

We denote the other rotations by $\rho^{2}$ and $\rho^{3}$ according to the usual notation. The rotation $\rho^{2}$ is also noted - since it can be seen as the complement morphism defined by the relations $\overline{\bar{a}}=a$ and $\overline{\bar{b}}=b$. Similarly, for $k \in\{0,1,2,3\}, \sigma_{k}$ is the reflection defined by the axis passing through the origin and having an angle of $k \pi / 4$ with the absciss. It may be seen as a morphism on $\mathcal{F}^{*}$ as well:

$$
\sigma_{0}: a \mapsto \bar{a}, \bar{a} \mapsto a, b \mapsto b, \bar{b} \mapsto \bar{b} \text { and } \sigma_{1}: a \mapsto b, b \mapsto a, \bar{a} \mapsto \bar{b}, \bar{b} \mapsto \bar{a}
$$

The two other reflections are $\sigma_{2}=\sigma_{0} \circ \rho^{2}$ and $\sigma_{3}=\sigma_{1} \circ \rho^{2}$. Another useful map is the antimorphism $\hat{\cdot}=\tau_{0} \sim$ defined on $\mathcal{F}^{*}$. If $w \in \mathcal{F}^{*}$ is a path, then $\widehat{w}$ is the path run in the opposite direction. The effect of the operators $\hat{\cdot}, \tilde{r}$ and $\bar{r}$ are illustrated in Figure 5 .

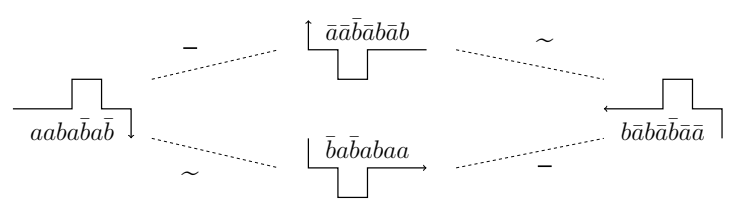

Fig. 5. Effect of the operators $\hat{\cdot}, \sim$ and $\bar{r}$ on $\mathcal{F}^{*}$

On the alphabet $\mathcal{R}$, we define an involution

$$
\imath: \mathrm{L} \mapsto \mathrm{R}, \mathrm{R} \mapsto \mathrm{L}, \mathrm{F} \mapsto \mathrm{F}, \mathrm{B} \mapsto \mathrm{B} .
$$

This function $\imath$ extends to $\mathcal{R}^{*}$ as a morphism, so that the map $\hat{\cdot}$ extends as well to $\hat{\cdot}: \mathcal{R}^{*} \rightarrow \mathcal{R}^{*}$ by $\hat{\cdot}=\imath \circ^{\sim}$. All these operations are closely related as shown in the lemmas hereafter. The proofs are left to the reader.

Lemma 2. Let $w \in \mathcal{F}^{*}, y \in \mathcal{R}^{*}$ and $\alpha \in \mathcal{F}$. The following properties hold:

(i) $\partial w=\partial \rho^{i}(w)$ for all $i \in\{1,2,3\}$,

(ii) $\imath(\partial w)=\partial \sigma_{i}(w)$ for all $i \in\{0,1,2,3\}$,

(iii) $\partial \widehat{w}=\widehat{\partial w}=\partial \widetilde{w}$,

(iv) $\rho^{i}\left(\int_{\alpha} y\right)=\int_{\rho^{i}(\alpha)} y$ for all $i \in\{1,2,3\}$,

(v) $\sigma_{i}\left(\int_{\alpha} y\right)=\int_{\sigma_{i}(\alpha)} \imath(y)$ for all $i \in\{0,1,2,3\}$,

(vi) $\widetilde{\int_{\alpha} y}=\int_{\beta} \widehat{y}$ where $\beta$ is the last letter of $\int_{\alpha} y$,

(vii) $\widehat{\int_{\alpha} y}=\int_{\bar{\beta}} \widehat{y}$ where $\beta$ is the last letter of $\int_{\alpha} y$,

(viii) If $\beta$ is the last letter of $\int_{\alpha} y$, then $\beta=\rho^{i}(\alpha)$ where $i=\Delta(y)$. 
For the rest of the paper, the words $w$ of $\mathcal{F}^{*}$ and $\mathcal{R}^{*}$ satisfying $\widehat{w}=w$ are called antipalindromes.

Lemma 3. Let $w \in \mathcal{F}^{*}$. Then the following statements are equivalent.

(i) $\widehat{w}=\rho^{2}(w)$

(ii) $w$ is a palindrome

(iii) $\partial w$ is an antipalindrome.

Finally, reflections on $\mathcal{F}^{*}$ are easily described on $\mathcal{R}^{*}$.

Lemma 4. Let $w \in \mathcal{F}^{*}$. There exists $i \in\{0,1,2,3\}$ such that $\widehat{w}=\sigma_{i}(w)$ if and only if $\partial w$ is a palindrome.

Square Tilings. Let $P$ be a polyomino having $W$ as a boundary word and $Q$ a square having $V=A B \widehat{A} \widehat{B}$ as a BN-factorization. Then the product of $P$ and $Q$, denoted by $P \circ Q$, is the polyomino whose boundary word is given by $\gamma(W)$, where $\gamma: \mathcal{F}^{*} \rightarrow \mathcal{F}^{*}$ is the morphism defined by $\gamma(a)=A, \gamma(\bar{a})=\widehat{A}, \gamma(b)=B$ and $\gamma(\bar{b})=\widehat{B}$. In 4 , a polyomino $R$ is called prime if for all pair of polyominoes $P$ and $Q$ such that $R=P \circ Q$, we have either $R=P$ or $R=Q$.

Proposition 1 (Provençal [4]). If a square $P$ has two factorizations, then they must alternate, i.e. no factor of one factorization is included in a factor of the other factorization.

Lemma 5. Let $P$ be a square of boundary word $W, A$ and $B$ be words such that $W \equiv A B \widehat{A} \widehat{B}$. Then $A, B \in \operatorname{Pal}\left(\mathcal{F}^{*}\right)$ if and only if $W=w \bar{w}$ for some word $w$.

Proof. If $W=w \bar{w}$ then every conjugate of $W$ has this form. Therefore, if $W \equiv$ $A B \widehat{A} \widehat{B}$, we have that $A B=\widehat{\widehat{A} \widehat{B}}=\widetilde{A} \widetilde{B}$, showing that $A$ and $B$ are palindromes. Conversely, one shows that if $A$ and $B$ are palindromes, then $W \equiv A B \overline{A B}$.

For more details on tiling by translation and square tilings see [110].

\section{Christoffel Tiles}

Recall that Christoffel words are finite Sturmian words, that is, they are obtained by discretizing a segment in the plane. Let $(p, q) \in$ $\mathbb{N}^{2}$ with $\operatorname{gcd}(p, q)=1$, and let $S$ be the segment with endpoints $(0,0)$ and $(p, q)$. The word $w$ is a lower Christoffel word if the path induced by $w$ is under $S$ and if they both delimit a polygon with no integral interior point. An

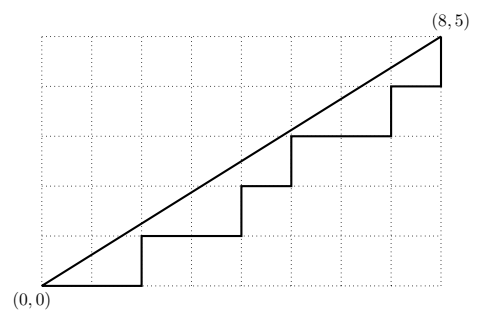
upper Christoffel word is defined similarly. A Christoffel word is either a lower 
Christoffel word or an upper Christoffel word. On the right is illustrated the lower one corresponding to

$$
w=a a b a a b a b a a b a b .
$$

It is well known that if $w$ and $w^{\prime}$ are respectively the lower and upper Christoffel words associated to $(p, q)$, then $w^{\prime}=\widetilde{w}$. Moreover, we have $w=a m b$ and $w^{\prime}=b m a$, where $m$ is a palindrome and $a, b$ are letters. We call cutting word the word $m$. They have been widely studied in the litterature (see e.g. [1], where they are also called central words . The next theorem gives a very useful characterization of Christoffel words.

Theorem 1 (Pirillo [12]). A word $m$ on the two-letter alphabet $\{a, b\} \subset \mathcal{F}$ is a cutting word if and only if amb and bma are conjugate.

Another useful result is the following.

Proposition 2 (Borel and Reutenauer [13]). The lower and upper Christoffel words $w$ and $w^{\prime}$ are conjugate by palindromes.

Let $\mathcal{B}=\{a, b\}$. Consider the morphism $\lambda: \mathcal{B}^{*} \rightarrow \mathcal{F}^{*}$ by $\lambda(a)=a \bar{b} a b$ and $\lambda(b)=a b$, which can be seen as a "crenellization" of the steps east and northeast. Two useful properties of $\lambda$ are used through the rest of this section and are easy to establish.

Lemma 6. Let $v, v^{\prime} \in \mathcal{B}^{*}$. Then

(i) $b \lambda(v)$ is a palindrome if and only if $v$ is a palindrome.

(ii) $\lambda(v) \equiv \lambda\left(v^{\prime}\right)$ if and only if $v \equiv v^{\prime}$.

We call crenellated tile a polyomino whose boundary word is given by $\lambda(w) \overline{\lambda(w)}$ where $w=a v b$ and $v \in \operatorname{Pal}\left(\mathcal{B}^{*}\right)$. A crenellated tile is a square since

$$
\lambda(w) \overline{\lambda(w)}=a \bar{b} a b \lambda(v) a b \bar{a} b \bar{a} \overline{b \lambda(v)} \bar{a} \bar{b} \equiv \bar{b} a \bar{b} \cdot a b \lambda(v) a \cdot \widehat{\bar{b} a \bar{b}} \cdot a \widehat{b \lambda(v)} a .
$$

We say that a crenellated tile obtained from a lower Christoffel word $w$ is a basic Christoffel tile while a Christoffel tile is a polyomino isometric to a basic Christoffel tile under some rotations $\rho$ and symmetries $\sigma_{i}$ (see Figure 6).
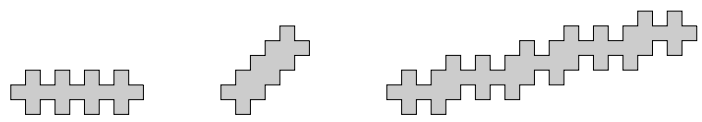

Fig. 6. Basic Christoffel tiles: (a) $w=a a a a b$ (b) $w=a b b b b$ and (c) $w=a a b a a b a b a a b a b$

Theorem 2. Let $P$ be a crenellated tile. Then $P$ is a double square if and only if it comes from a Christoffel word. 
Proof. $(\Rightarrow)$ Assume that $P$ is a double square. Let $W=\lambda(w) \overline{\lambda(w)}$ be its boundary word, where $w=a v b \in a \operatorname{Pal}\left(\mathcal{B}^{*}\right) b$. We know from Proposition 1 that the factorizations must alternate. Since $P$ has the factorization

$$
W=\bar{b} a \bar{b} \cdot a b \lambda(v) a \cdot \widehat{\bar{b} a \bar{b}} \cdot \widehat{a b \lambda(v)} a
$$

it means that the second factorization is obtained from one of the conjugates $W^{\prime}=a \bar{b} a b \lambda(v) a b \bar{a} b \bar{a} \bar{b} \lambda(v) \bar{a} \bar{b}$ or $W^{\prime \prime}=\bar{b} a b \lambda(v) a b \bar{a} b \bar{a} \bar{b} \lambda(v) \bar{a} \bar{b} a$. Let $V^{\prime}$ and $V^{\prime \prime}$ be respectively the first half of $W^{\prime}$ and $W^{\prime \prime}$. Then, by Lemma [5] either $V^{\prime}$ or $V^{\prime \prime}$ is a product of two palindromes $x$ and $y$. First, assume that the other factorization is obtained from $V^{\prime}$. Then $V^{\prime}=\lambda(a v b)=a \bar{b} a b \lambda(v) a b=x y$. Taking the reversal on each side, we get $\widehat{\lambda(a v b)}=y x$, that is $\lambda(a v b) \equiv \widehat{\lambda(a v b)}$. But

$$
\widetilde{\lambda(a v b)}=b a \widetilde{\lambda(v)} b a \bar{b} a=b a \widehat{b \lambda(v)} a \bar{b} a=b a b \lambda(v) a \bar{b} a \equiv a b \lambda(v) a \bar{b} a b=\lambda(b v a),
$$

which means that $\lambda(a v b) \equiv \lambda(b v a)$. Thus, by Lemma 6, we deduce $a v b \equiv b v a$. Hence, by Theorem 1 we conclude that $v$ is a cutting word so that $w=a v b$ is a lower Christoffel word.

It remains to consider the case where the second factorization is obtained from $V^{\prime \prime}$. Hence, we could write $V^{\prime \prime}=\bar{b} a b \lambda(v) a b \bar{a}=x y$. But such palindromes $x$ and $y$ cannot exist since $\bar{a}$ appears only at the end of $V^{\prime \prime}$.

$(\Leftarrow)$ Assume that $w$ is a lower Christoffel word. We know from Proposition 2 that $w=a m a b m^{\prime} b$ for some palindromes $m$ and $m^{\prime}$. Therefore,

$$
\begin{aligned}
\lambda(w) \overline{\lambda(w)} & =\lambda\left(a m a b m^{\prime} b\right) \overline{\lambda\left(a m a b m^{\prime} b\right)} \\
& =a \bar{b} a b \lambda(m) a \bar{b} a \cdot b a b \lambda\left(m^{\prime}\right) a b \cdot \bar{a} b \bar{a} \bar{b} \overline{\lambda(m)} \bar{a} b \bar{a} \cdot \bar{b} \bar{a} \bar{b} \overline{\lambda\left(m^{\prime}\right)} \bar{a} \bar{b}
\end{aligned}
$$

showing that $P$ admits a second square factorization.

It can also be shown in view of Lemma [5] that for each Christoffel tile such that $W \equiv A B \widehat{A} \widehat{B}$, the factors $A$ and $B$ are palindromes which suggests that the conjecture of Provençal [4] is true.

\section{Fibonacci Tiles}

In this section, in order to simplify the notation, we overload the operator - by defining it over $\mathcal{R}^{*}$ by $\bar{y}=\iota(y)$ for all $y \in \mathcal{R}^{*}$. We define a sequence $\left(q_{n}\right)_{n \in \mathbb{N}}$ in $\mathcal{R}^{*}$ by $q_{0}=\varepsilon, q_{1}=\mathrm{R}$ and

$$
q_{n}= \begin{cases}q_{n-1} q_{n-2} & \text { if } n \equiv 2 \quad \bmod 3 \\ q_{n-1} \overline{q_{n-2}} & \text { if } n \equiv 0,1 \quad \bmod 3\end{cases}
$$

whenever $n \geq 2$. The first terms of $\left(q_{n}\right)_{n \in \mathbb{N}}$ are

$$
\begin{array}{lll}
q_{0}=\varepsilon & q_{3}=\mathrm{RL} & q_{6}=\text { RLLRLLRR } \\
q_{1}=\mathrm{R} & q_{4}=\mathrm{RLL} & q_{7}=\text { RLLRLLRRLRRLR } \\
q_{2}=\mathrm{R} & q_{5}=\mathrm{RLLRL} & q_{8}=\text { RLLRLLRRLRRLRRLLRLLRR }
\end{array}
$$


Note that $\left|q_{n}\right|=F_{n}$ is the $n$-th Fibonacci number. Moreover, given $\alpha \in \mathcal{F}$, the path $\int_{\alpha} q_{n}$ presents strong symmetric properties, as shown by the next lemma.

Lemma 7. Let $n \in \mathbb{N}$. Then $q_{3 n+1}=p \alpha, q_{3 n+2}=q \alpha$ and $q_{3 n+3}=r \bar{\alpha}$ for some antipalindrome $p$, and some palindromes $q, r$ and some letter $\alpha \in\{\mathrm{L}, \mathrm{R}\}$.

Proof. By induction on $n$. For $n=0$, we have indeed $q_{1}=\varepsilon \cdot \mathrm{R}, q_{2}=\varepsilon \cdot \mathrm{R}$ and $q_{3}=\mathrm{R} \cdot \mathrm{L}$. Now, assume that $q_{3 n+1}=p \alpha, q_{3 n+2}=q \alpha$ and $q_{3 n+3}=r \bar{\alpha}$ for some antipalindrome $p$, some palindromes $q, r$ and some letter $\alpha \in\{\mathrm{L}, \mathrm{R}\}$. Then

$$
\begin{aligned}
& q_{3 n+4}=q_{3 n+3} \overline{q_{3 n+2}}=q_{3 n+2} \overline{q_{3 n+1} q_{3 n+2}}=q \alpha \overline{p \alpha q} \cdot \bar{\alpha} \\
& q_{3 n+5}=q_{3 n+4} q_{3 n+3}=q_{3 n+3} \overline{q_{3 n+2}} q_{3 n+3}=r \overline{\alpha q \alpha} r \cdot \bar{\alpha} \\
& q_{3 n+6}=q_{3 n+5} \overline{q_{3 n+4}}=q_{3 n+4} q_{3 n+3} \overline{q_{3 n+4}}=q \alpha \overline{p \alpha q \alpha} r \overline{\alpha q \alpha} p \alpha q \cdot \alpha .
\end{aligned}
$$

Since $q \alpha \overline{p \alpha q}$ is an antipalindrome and $r \overline{\alpha q \alpha} r, q \alpha \overline{p \alpha q \alpha} r \overline{\alpha q \alpha} p \alpha q$ are palindromes, the result follows.

The proof of the following lemma is technical and can be done by induction. It is not included here due to lack of space.

Lemma 8. Let $n \in \mathbb{N}$ and $\alpha \in \mathcal{F}$.

(i) The path $\int_{\alpha} q_{n}$ is simple.

(ii) The path $\oint_{\alpha}^{\alpha}\left(q_{3 n+1}\right)^{4}$ is the boundary word of a polyomino.

A Fibonacci tile of order $n$ is a polyomino having $\oint_{\alpha}\left(q_{3 n+1}\right)^{4}$ as a boundary word, where $n \in \mathbb{N}$. They are somehow related to the Fibonacci Fractals found in [14. The first Fibonacci tiles are illustrated in Figure 7
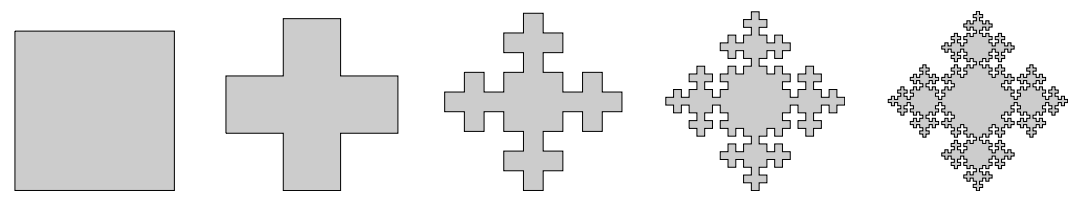

Fig. 7. Fibonacci tiles of order $n=0,1,2,3,4$

Theorem 3. Fibonacci tiles are double squares.

Proof. We know from Lemma 7 that $q_{3 n+1}=p x$ for some antipalindrome $p$ and some letter $x \in\{\mathrm{L}, \mathrm{R}\}$. If $x=\mathrm{R}$, we consider the reversal of the path, i.e. $\widehat{\left(q_{3 n+1}\right)^{4}}$, so that we may suppose that $x=\mathrm{L}$. Therefore, on the first hand, we obtain

$$
\oint_{\alpha}\left(q_{3 n+1}\right)^{4}=\int_{\alpha} p \mathrm{~L} \cdot p \mathrm{~L} \cdot \widehat{p} \mathrm{~L} \cdot \widehat{p}=\int_{\alpha} p \cdot \int_{\rho(\alpha)} p \cdot \widehat{\int_{\alpha}} p \cdot \widehat{\int_{\rho(\alpha)}} p,
$$


because $\Delta(p)=0$. On the other hand, the conjugate $q_{3 n+1}^{\prime}=\overline{q_{3 n-1}} q_{3 n}$ of $q_{3 n+1}$ corresponds to another boundary word of the same tile. Using again Lemma 7 . we can write $q_{3 n}=r \mathrm{~L}$ and $q_{3 n-1}=q \mathrm{R}$, for some palindromes $q$ and $r$. Therefore, $p \mathrm{~L}=q_{3 n+1}=q_{3 n} \overline{q_{3 n-1}}=r \mathrm{~L} \bar{q} \mathrm{~L}$ so that $p=r \mathrm{~L} \bar{q}$. But $p$ is an antipalindrome, which means that $q_{3 n+1}^{\prime}=\overline{q_{3 n-1}} q_{3 n}=\bar{q} \mathrm{~L} r \mathrm{~L}=\widetilde{p} \mathrm{~L}=\bar{p} \mathrm{~L}$. Hence, since $\bar{p}$ is an antipalindrome as well, we find

$$
\oint_{\alpha}\left(q_{3 n+1}^{\prime}\right)^{4}=\int_{\alpha} \bar{p} \mathrm{~L} \cdot \bar{p} \mathrm{~L} \cdot \widehat{\bar{p}} \mathrm{~L} \cdot \widehat{\bar{p}}=\int_{\alpha} \bar{p} \cdot \int_{\rho(\alpha)} \bar{p} \cdot \widehat{\int_{\alpha}} \bar{p} \cdot \widehat{\int_{\rho(\alpha)}} \bar{p} .
$$

As for Christoffel Tiles, Fibonacci Tiles also suggest that the conjecture of Provençal for double squares 4 is true as stated in the next result.

Corollary 1. If $A B \widehat{A} \widehat{B}$ is a $B N$-factorisation of a Fibonacci tile, then $A$ and $B$ are palindromes.

Proof. The conclusion follows from Lemmas 2 and 3 and Theorem 3 . Indeed, since $p$ is an antipalindrome, then $\int_{\alpha} p$ is a palindrome. The same argument applies for the second factorization.

We end this section by presenting four families of double squares generalizing the Fibonacci tiles. Consider the sequence $\left(r_{d, m, n}\right)_{(d, m, n) \in \mathbb{N}^{3}}$ satisfying the following recurrence, for $d \geq 2$,

$$
r_{d, m, n}= \begin{cases}r_{d-1, n, m} \overline{r_{d-2, n, m}} & \text { if } d \equiv 0 \bmod 3 \\ r_{d-1, n, m} r_{d-2, n, m} & \text { if } d \equiv 1 \bmod 3 \\ r_{d-1, m, n} \overline{r_{d-2, m, n}} & \text { if } d \equiv 2 \bmod 3\end{cases}
$$

Using similar arguments as in the Fibonacci tiles case, one shows that both families obtained respectively with seed values

$$
\begin{aligned}
r_{0, m, n} & =(\mathrm{RLLR})^{m} \mathrm{RLR}, & r_{1, m, n} & =(\mathrm{RLLR})^{n} \mathrm{R}, \\
r_{0, m, n} & =(\mathrm{RL})^{m} \mathrm{RLR}, & r_{1, m, n} & =(\mathrm{RL})^{n} \mathrm{RL}
\end{aligned}
$$

are such that $\oint_{\alpha}\left(r_{d, m, n} r_{d, n, m}\right)^{2}$ is a boundary word whose associated polyomino is a double square (see Figure 8), where $\alpha \in \mathcal{F}$. Their level of fractality increases with $d$ so that one could say that they are crenellated versions of the Fibonacci Tiles.

Similarly, let $\left(s_{d, m, n}\right)_{(d, m, n) \in \mathbb{N}^{3}}$ be a sequence satisfying for $d \geq 2$ the recurrence

$$
s_{d, m, n}= \begin{cases}s_{d-1, n, m} \overline{s_{d-2, n, m}} & \text { if } d \equiv 0,2 \bmod 3, \\ s_{d-1, m, n} s_{d-2, m, n} & \text { if } d \equiv 1 \bmod 3 .\end{cases}
$$

Then the families obtained with seed values

$$
\begin{array}{ll}
s_{0, m, n}=(\mathrm{RLLR})^{m} \mathrm{RLR}, & s_{1, m, n}=\mathrm{RL}, \\
s_{0, m, n}=(\mathrm{RL})^{m} \mathrm{RLR}, & s_{1, m, n}=\mathrm{R}
\end{array}
$$

yield double squares $\oint_{\alpha}\left(s_{d, m, n} s_{d, n, m}\right)^{2}$ as well (see Figure 9). One may verify that $r_{d, 0,0}=s_{d, 0,0}$ for any $d \in \mathbb{N}$ if the seed values are respectively (2a) and (3b) or respectively (2b) and (3a). 

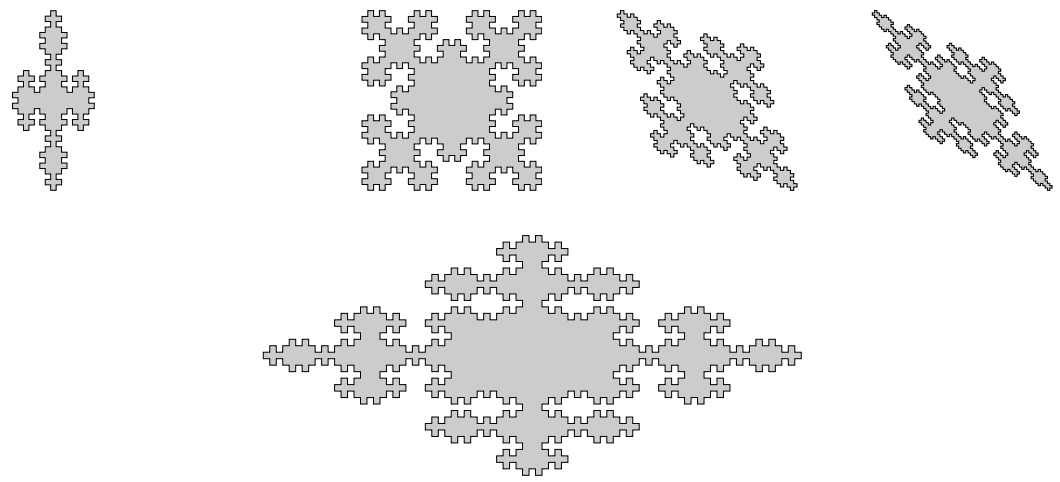

Fig. 8. Upper row: tile obtained from $r_{2,0,1}$ with seeds (2a); tiles obtained from $r_{3, m, 0}$ with seeds (2b), for $m=0,1,2$. Lower row: generalized Fibonacci tile obtained with parameters $d=3, m=1$ and $n=0$ on sequence $r$ with seed values (2a).
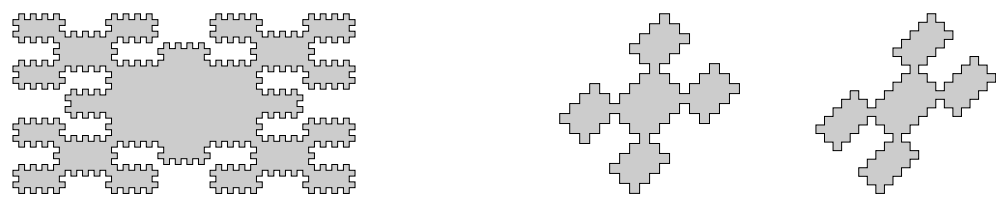

Fig. 9. Tile obtained from $s_{3,2,0}$ with seeds (3a); tiles obtained from $s_{2,0, n}$ with seeds (3b) for $n=1,2$

\section{Concluding Remarks}

The study of double squares suggests interesting and challenging problems. For instance, it is appealing to conjecture that a prime double square is either of Christoffel type or of Fibonacci type. However, that is not the case, as illustrated by Figure 10. This begs for a thorough study in order to exhibit a complete zoology of such tilings. On the other hand, there is a conjecture of 4 stating that if $A B \widehat{A} \widehat{B}$ is the BN-factorization of a prime double square, then $A$ and $B$ are palindromes, for which we have not been able to provide any counterexample. Another problem is to prove that Christoffel and Fibonacci tiles are prime, that is, they are not obtained by composition of smaller squares. This
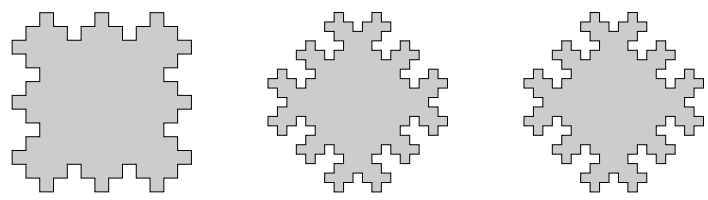

Fig. 10. Three double squares not in the Christoffel and Fibonacci tiles families 
leads to a number of questions on the "arithmetics" of tilings, such as the unique decomposition, distribution of prime tiles, and their enumeration.

The fractal nature of the Fibonacci tiles strongly suggests that Lindemayer systems ( $L$-systems) may be used for their construction [15]. The formal grammars used for describing them have been widely studied, and their impact in biology, computer graphics [16] and modeling of plants is significant [17]. A number of designs including snowflakes fall into this category.

\section{References}

1. Brlek, S., Provençal, X.: An optimal algorithm for detecting pseudo-squares. In: Kuba, A., Nyúl, L.G., Palágyi, K. (eds.) DGCI 2006. LNCS, vol. 4245, pp. 403-412. Springer, Heidelberg (2006)

2. Wijshoff, H.A.G., van Leeuven, J.: Arbitrary versus periodic storage schemes and tesselations of the plane using one type of polyomino. Inform. Control 62, 1-25 (1984)

3. Beauquier, D., Nivat, M.: On translating one polyomino to tile the plane. Discrete Comput. Geom. 6, 575-592 (1991)

4. Provençal, X.: Combinatoire des mots, géométrie discrète et pavages. PhD thesis, D1715, Université du Québec à Montréal (2008)

5. Brlek, S., Labelle, G., Lacasse, A.: Properties of the contour path of discrete sets. Int. J. Found. Comput. Sci. 17(3), 543-556 (2006)

6. Lothaire, M.: Combinatorics on Words. Cambridge University Press, Cambridge (1997)

7. Freeman, H.: On the encoding of arbitrary geometric configurations. IRE Trans. Electronic Computer 10, 260-268 (1961)

8. Freeman, H.: Boundary encoding and processing. In: Lipkin, B., Rosenfeld, A. (eds.) Picture Processing and Psychopictorics, pp. 241-266. Academic Press, New York (1970)

9. Braquelaire, J.P., Vialard, A.: Euclidean paths: A new representation of boundary of discrete regions. Graphical Models and Image Processing 61, 16-43 (1999)

10. Brlek, S., Provençal, X., Fédou, J.M.: On the tiling by translation problem. Discrete Applied Mathematics 157(3), 464-475 (2009)

11. Berstel, J., Lauve, A., Reutenauer, C., Saliola, F.: Combinatorics on Words: Christoffel Words and Repetition in Words. CRM monograph series, vol. 27, 147 pages. American Mathematical Society, Providence (2008)

12. Pirillo, G.: A new characteristic property of the palindrome of standard sturmian word. Sém. Lothar. Combin. 43, 1-3 (1999)

13. Borel, J.P., Reutenauer, C.: On christoffel classes. RAIRO-Theoretical Informatics and Applications 40, 15-28 (2006)

14. Monnerot-Dumaine, A.: The Fibonacci fractal. Submitted (September 2008)

15. Rozenberg, G., Salomaa, A.: Mathematical Theory of L Systems. Academic Press, Inc., Orlando (1980)

16. Rozenberg, G., Salomaa, A. (eds.): Lindenmayer Systems: Impacts on Theoretical Computer Science, Computer Graphics, and Developmental Biology. Springer, Secaucus (2001)

17. Prusinkiewicz, P., Lindenmayer, A.: The algorithmic beauty of plants. Springer, New York (1990) 